\title{
Prevention of Gestational Diabetes in Pregnant Women with Obesity: Protocol for a Pilot Randomised Controlled Trial
}

Ola Quotah

King's College London https://orcid.org/0000-0002-8883-3300

Glen Nishku

King's College London

Jessamine Hunt

King's College London

Paul T Seed

King's College London

Carolyn Gill

King's College London

Anna Brockbank

King's College London

Omoyele Fafowora

King's College London

llektra Vasiloudi

King's College London

Opeoluwa Olusoga

King's College London

Ellie Cheek

King's College London

Jannelle Phillips

King's College London

Katarzyna G Nowak

King's College London

\section{Lucilla Poston}

King's College London Faculty of Life Sciences and Medicine

\section{Sara L White}

King's College London Faculty of Life Sciences and Medicine Angela C Flynn ( $\square$ angela.flynn@kcl.ac.uk)

King's College London Faculty of Life Sciences and Medicine 


\section{Research Article}

Keywords: Gestational diabetes, maternal obesity, lifestyle intervention, metformin

Posted Date: September 30th, 2021

DOl: https://doi.org/10.21203/rs.3.rs-924115/v1

License: (c) (i) This work is licensed under a Creative Commons Attribution 4.0 International License. Read Full License

Version of Record: A version of this preprint was published at Pilot and Feasibility Studies on March 25th, 2022. See the published version at https://doi.org/10.1186/s40814-022-01021-3. 


\section{Abstract}

\section{Background}

Obesity in pregnancy increases the risk of gestational diabetes mellitus (GDM) and associated adverse outcomes. Despite metabolic differences, all pregnant women with obesity are considered to have the same risk of developing GDM. Improved risk stratification is required to enable targeted intervention in women with obesity who would benefit the most. The aim of this study is to identify pregnant women with obesity at higher risk of developing GDM and in a pilot randomised controlled trial (RCT), show efficacy of a lifestyle intervention and/or metformin to improve glycaemic control.

\section{Methods}

Women aged 18 years or older with a singleton pregnancy and body mass index $(\mathrm{BMI}) \geq 30 \mathrm{~kg} / \mathrm{m}^{2}$ will be recruited from one maternity unit in London, UK. Risk of GDM will be assessed using a multivariable GDM prediction model combining maternal age, mid-arm circumference, systolic blood pressure, serum triglycerides and whole blood $\mathrm{HbA} 1 \mathrm{c}$. Women identified at higher risk of developing GDM will be randomly allocated to one of two intervention groups (lifestyle advice with or without metformin) or standard antenatal care. Primary outcome; reduction in mean glucose $/ 24 \mathrm{hr}$ of $0.5 \mathrm{mmol} / \mathrm{l}$ as assessed by continuous glucose monitoring. A sample of 60 high risk women is required.

\section{Discussion}

Early risk stratification of GDM in pregnant women with obesity and targeted intervention using lifestyle advice with or without metformin could improve glucose tolerance compared to standard antenatal care. If successful, an adequately powered RCT will be performed to investigate whether this strategy is effective in preventing GDM in women with obesity.

\section{Ethics and trial registration}

This study has been approved by the NHS Research Ethics Committee (UK IRAS integrated research application system; reference 18/LO/1500). EudraCT Number 2018-000003-16.

\section{Introduction}

Global estimates suggest that over $21 \%$ of women will be obese by 2025 [1]. In the UK, over $20 \%$ of women present with obesity at their first antenatal visit [2]. Obesity adversely affects reproductive health [3]. Pregnant women with obesity have a higher risk of developing most pregnancy complications, including gestational diabetes mellitus (GDM) [4, 5], and women with GDM are more likely to develop hypertension and pre-eclampsia during pregnancy $[6,7]$ as well as cardiovascular disease and type 2 diabetes later in life [8-10]. Infants born to mothers with GDM are more likely to be born large-forgestational age (LGA) and macrosomic, increasing the risk of complications at delivery, including 
shoulder dystocia [7, 11]. Furthermore, offspring of GDM mothers have a higher risk of being overweight or obese [12], developing diabetes $[13,14]$ or metabolic disease $[15,16]$ later in life.

UK guidelines recommend that all pregnant women with obesity undergo an oral glucose tolerance test (OGTT) at 24-28 weeks' gestation to detect GDM and introduce treatment [17]. Evidence suggests, however, that excessive fetal growth precedes the time of diagnosis in obese women [18]. Furthermore, marked abnormalities in the metabolic profiles of obese women who develop GDM may occur at least ten weeks before conventional diagnosis $[19,20]$.

Several randomised controlled trials (RCTs) have attempted to prevent adverse outcomes in obese women; diet and physical activity [21] or pharmacological interventions [22-24] have overall not been effective. This has shifted the focus to targeted intervention for those individuals identified at greatest risk and increasing evidence suggests that this approach might be beneficial. The RADIEL RCT of a lifestyle intervention in 293 Finnish pregnant women, of whom $30 \%$ had previous GDM, demonstrated a reduction in GDM from 21.6-13.9\% [25]. This observation was further supported by a UK multicentre randomised trial (ESTEEM) of a Mediterranean-style diet in women with risk factors including obesity, chronic hypertension or hypertriglyceridaemia, in which a reduction in GDM of $35 \%$ in the intervention group was achieved [26].

Although several studies describe tools developed to predict GDM in weight heterogenous women [2733], specific approaches for pregnant women with obesity are rare. At present, all pregnant women with a body mass index $(\mathrm{BMI}) \geq 30 \mathrm{~kg} / \mathrm{m}^{2}$ are considered at high risk of developing GDM, although, the majority do not develop the condition [34]. Our prediction tool for GDM was constructed using a range of biochemical and clinical factors in early pregnancy obtained in a cohort of 1303 obese pregnant women who took part in the UK Pregnancies Better Eating and Activity Trial (UPBEAT) [34]. The tool was developed using the clinical variables maternal age, blood pressure and mid-arm circumference and the biomarkers $\mathrm{HbA} 1 \mathrm{c}$ and serum triglycerides. At a threshold of $\geq 35 \%$ risk, the tool identifies in early pregnancy obese pregnant women with a higher risk of developing GDM ( 1 in 2 chance) when diagnosed using The International Association of Diabetes and Pregnancy Study Groups (IADPSG) criteria, and thereby those who might benefit most from an intervention [35].

In this, the next step, a pilot study will be undertaken in which pregnant women with obesity and at higher risk of developing GDM as identified using the prediction tool will be randomised to one of two interventions designed to improve glucose tolerance, or to standard antenatal care.

\section{Study objectives}

The primary objective of the UK Pregnancies Better Eating and Activity Trial-Taking It Forward (UPBEATTIF) study is to assess in pregnant women with obesity, identified as high risk for GDM, the efficacy of a) lifestyle advice (diet and physical activity), b) metformin treatment plus lifestyle advice, to improve maternal glycaemic control, when compared with c) standard care. A secondary objective is to determine 
the impact of each intervention on a targeted maternal metabolome. Further objectives are to examine the effect of each intervention on dietary intake and physical activity.

\section{Methods And Analysis}

This protocol paper is written in accordance with the Standard Protocol Items: Recommendations for Interventional Trials (SPIRIT) checklist [36].

\section{Study design}

This study will be a single centre, open-label, randomised controlled trial. NHS Research Ethics Committee approval has been obtained (UK IRAS integrated research application system; reference 18/LO/1500).

\section{Study setting}

Women will be recruited in one centre, from the antenatal clinics of Guy's and St Thomas' NHS Foundation Trust, London, UK (Figure 1).

\section{Target population}

Pregnant women with obesity will be invited to be screened for risk of developing GDM at their routine nuchal ultrasound scan appointment at St Thomas' Hospital. Women will be eligible to take part in the study if they meet the following criteria:

- Over 18 years

- $\mathrm{BMI} \geq 30 \mathrm{~kg} / \mathrm{m}^{2}$

- Higher risk of GDM as identified by risk assessment at screening

- Singleton pregnancy

- Gestation $11-14^{+6}$ weeks' at the time of screening

- Willing and able to give written informed consent

\section{Exclusion criteria}

Women will be excluded if they meet the following criteria:

- Women identified as being at background risk of GDM at screening

- Taking metformin or any medications that affect insulin sensitivity

- $\mathrm{HbA1c} \geq 6.5 \%$ at the time of screening

- Pre-existing medical conditions including diabetes, thyroid disease, coeliac disease, hypertension, sickle cell, systemic lupus erythematosus, antiphospholipid syndrome, thalassaemia, current psychosis

- Past bariatric surgery 
- Multiple pregnancy

- Insufficient understanding of the trial

- Contraindications to metformin

- Participation in another investigational medicinal product trial at the time of screening

\section{Study entry}

\section{$11-14^{+6}$ weeks' gestation appointment: screening}

Eligible women will be contacted and offered information about the study before their routine nuchal ultrasound scan appointment in the antenatal clinic of St Thomas' Hospital. Eligible women will also be approached in the antenatal clinic, given information about the study and offered the prediction test to identify risk of later GDM development. Following informed written consent, weight will be measured, and a blood sample taken at the same time as routine blood sampling for measurement of $\mathrm{HbA} 1 \mathrm{c}$ and triglycerides. Clinical risk factors will be also recorded (age, blood pressure, mid-arm circumference) for risk assessment. Risk will be assessed by the algorithm previously described [35].

All women screened will be contacted by telephone. Women identified as being at background risk of developing GDM will be managed according to clinical guidelines and will not enter the trial. Women identified as being at a higher risk will be invited to be randomised into an antenatal programme of lifestyle advice, lifestyle advice plus metformin treatment or standard antenatal care.

\section{$13-15^{+6}$ weeks' gestation appointment: baseline and randomisation}

At the first appointment, informed consent will be obtained from women to be randomised into the trial. All participants will be fitted with a glucose sensor (Dexcom G6) to be worn for 7 days. The sensors are small, easy to wear, waterproof devices which require no finger prick calibration. The sensors will be applied to the back of the upper arm or abdomen of the participant and activated in blinded mode. To assess dietary intake, a semi-quantitative food frequency questionnaire (FFQ) will be administered to evaluate dietary glycaemic load (GL), glycaemic index (GI), saturated fat and other dietary variables [34]. Physical activity will be assessed by Axivity accelerometer AX3 (https://axivity.com/product/ax3).

Following the baseline assessment, randomisation will be performed via a secure online-based data management platform (MedSciNet $\left.{ }^{\mathrm{TM}}\right)$. Women will be randomised into three groups:
a. Lifestyle advice
b. Lifestyle advice plus metformin
c. Standard antenatal care

The randomization schedule will be minimized according to ethnicity (Black, White, Asian, Other), BMI $\left(30-34.9 \mathrm{~kg} / \mathrm{m}^{2}, 35-39.9 \mathrm{~kg} / \mathrm{m}^{2},{ }^{3} 40 \mathrm{~kg} / \mathrm{m}^{2}\right)$, parity (nulliparous/multiparous) and age. Participants will be allocated sequential study numbers regardless of allocation to the intervention or standard care group. 
Due to the nature of the study design, it will not be possible to blind participants or research staff to the randomisation arm.

\section{Intervention}

\section{Lifestyle advice}

Participants allocated to the lifestyle advice or lifestyle advice plus metformin arms will receive the intervention. The dietary intervention will focus on reducing $\mathrm{GL}$ and saturated fat intake using a regime previously shown to be effective in changing diet in 'all risk' obese pregnant women [34]. The dietary component of the intervention will aim to promote a healthier pattern of eating without restricting energy intake. The dietary advice will be tailored according to the woman's habitual diet and cultural preference.

To decrease GL, participants will be encouraged to exchange starchy foods with a medium to high GI for those with a lower $\mathrm{Gl}$ and reduce the consumption of sugar sweetened beverages including fruit juice. Specific dietary goals will be provided for each participant that focus on differentiating between carbohydrate rich foods. This advice will include replacing high $\mathrm{GI}$ breads with low $\mathrm{Gl}$ breads such as granary/wholegrain/multigrain bread, sourdough, rye; replacing high GI breakfast cereals with low GI varieties such as porridge, oat-based cereals, no-added sugar muesli; encouraging consumption of low GI grains like basmati rice, pearl barley, cracked wheat (bulgur), quinoa and pasta. To reduce saturated fat intake, participants will be encouraged to; swap oils and spreads high in saturated fats (butter, lard, ghee) for those high in mono- and polyunsaturated fats (olive, sunflower); swap meat high in saturated fat (fatty meats, processed meat and meat products) for lean red meat, chicken and fish; swap high fat dairy foods (milk, cheese, yogurt) for lower fat varieties.

The physical activity component of the intervention will focus on a safe physical activity regime using the UPBEAT strategy which shown to be successful in improving physical activity in 'all risk' pregnant women with obesity [34]. The advice aims to increase incremental walking and daily step count at a moderate intensity, being more active in daily life and reduce sedentary behaviour (sitting and screen time).

\section{Metformin}

In participants randomised to the lifestyle intervention plus metformin arm, metformin treatment will be initiated ( $500 \mathrm{mg}$ daily with food) and increased by $500 \mathrm{mg}$ every 2 to 3 days to achieve $2000 \mathrm{mg}$ within two weeks, taken as divided doses. Should side-effects occur, participants will be advised to reduce the current dose to that of the previous week and wait for 1 week before increasing the dose again. Treatment will be initiated after randomisation and continued until delivery of the baby. Metformin will be dispensed at study visits and compliance assessed by tablet count.

Participants randomised to the lifestyle intervention arms will receive weekly sessions for 8 consecutive weeks. The sessions will be covered by video, email or telephone. Following a general review of the lifestyle or lifestyle plus metformin intervention, each session will be designed to deliver a different element of the intervention. Development of the UPBEAT lifestyle intervention was informed by 
psychological models of health behaviour, including control and social cognitive theory [34]. Participants will be encouraged to set achievable goals using the SMART (Specific, Measurable, Achievable, Relevant, Time Specific) goal approach, which will be reviewed on a weekly basis. In addition, participants will be asked to self-monitor their progress using a logbook. The participants will also receive a handbook with guidance on making the changes, along with recipe ideas and general information on eating while pregnant.

\section{Standard Care}

Women randomised to the standard care group will attend all study visits except the 8 weekly intervention visits and receive routine antenatal care according to local health care provision.

\section{2-25 weeks' gestation appointment}

All participants will be fitted with a continuous glucose monitoring (CGM) sensor for 7 days, again in blinded mode. Dietary intake and physical activity will be assessed using the FFQ and accelerometer. In the 'lifestyle plus metformin' arm, metformin will be dispensed, information on any side effects or adverse events will be collected and compliance will be assessed by tablet count.

\section{4-28 weeks' gestation appointment}

All participants will be asked to attend at 24-28 week's gestation for an oral glucose tolerance test (fasting for a minimum of 10 hours, $75 \mathrm{~g}$ glucose load). Routine blood samples will be taken fasted and 2 hours after the glucose load in accordance with The National Institute of Clinical Excellence (NICE) guidelines [17]. Research blood samples will be obtained in the fasted state, 1 hour and 2 hours following the glucose load. GDM will be diagnosed in accordance with IADPSG criteria: fasting capillary glucose $\geq 5.1 \mathrm{mmol} / \mathrm{L}$ and/or 1 -hour glucose $\geq 10 \mathrm{mmol} / \mathrm{L}$ and/or 2-hour glucose $\geq 8.5 \mathrm{mmol} / \mathrm{L}$ [37]. If a participant develops GDM during the study, she will be asked to continue with the medication, but she will be referred to the antenatal diabetes team for further advice and treatment. Weight will be measured at this visit.

\section{4-36 weeks' gestation appointment}

Metformin will be dispensed to those allocated the drug. Women will be instructed to stop trial medication on the day of delivery and will be asked to hand in the rest of the trial medication to the research team. Information on any side effects and adverse events will be collected in the metformin arm. Compliance will be assessed by tablet count. Information on pregnancy complications will be collected and weight will be recorded in all arms.

\section{Trial outcomes}

The primary endpoint is a reduction in mean glucose $/ 24 \mathrm{hr}$ of $0.5 \mathrm{mmol} / \mathrm{l}$ after 8 -weeks of treatment as assessed using CGM.

Secondary outcome measures include: 
- Other glycaemic parameters after 8-weeks of treatment; mean daytime glucose $0.700-23.00 \mathrm{~h}$, mean nocturnal glucose $23.00-0.700 \mathrm{~h}$, percentage time in tight glucose control target $3.5-6.7 \mathrm{mmol} / \mathrm{L}$, percentage time in recommended glucose control target $3.5-7.8 \mathrm{mmol} / \mathrm{L}$, area under the curve (AUC) $<6.7 \mathrm{mmol} / \mathrm{L}, \mathrm{AUC}<7.8 \mathrm{mmol} / \mathrm{L}$, glucose variability measures (standard deviation (SD), coefficient of variation (CV)), High Blood glucose index (HBGI), Low Blood glucose index (LBGI).

- GDM diagnosis at 24-28 weeks' (in accordance with IADPSG and NICE criteria).

- Metabolome; a targeted high throughput nuclear magnetic resonance (NMR) metabolomic platform (http://computationalmedicine.fi/) will be used to measure metabolites from blood samples at baseline and at the time of the diagnostic OGTT. The platform quantifies lipid measures including lipoprotein particles (VLDL subdivided into 6 subclasses, IDL, LDL subdivided into 3 subclasses and HDL subdivided into 4 subclasses), constituents within each lipoprotein particle type (triglycerides, total cholesterol, free cholesterol and cholesterol ester levels and phospholipid concentrations), fatty acids, amino acids, glycolysis related metabolites, ketone bodies and inflammatory markers.

- Dietary intake and physical activity; including changes in $\mathrm{Gl}, \mathrm{GL}$, saturated fat, time spent in light and moderate exercise and sedentary behaviour from baseline and after the 8-week intervention.

\section{Sample size calculation and loss to follow-up}

Twenty participants per arm was calculated to be adequate to provide $>90 \%$ power to detect a $0.5 \mathrm{mmol} / \mathrm{I}$ difference in mean $24 \mathrm{hr}$ glucose between arms. This difference was considered to be of biological relevance based on data from comparable studies of CGM in pregnant women with obesity [38-40]. To account for $25 \%$ loss to follow up, a sample size of 84 women will be recruited.

\section{Statistical analysis}

An intention to treat analysis will be completed for participants in all arms. Demographic characteristics will be compared between groups using Student's t test or Mann-Whitney tests for continuous data and Pearson's chi-squared test for categorical data as appropriate. For continuous variables, results will be presented as means and standard deviations. The number and percentage will be presented for categorical and binary variables. Following distributional checks, log transformations will be carried out as needed. For the primary and main secondary glycaemic outcomes, an adjustment will be made using multiple linear regression (method analysis of covariance; ANCOVA [41]). For yes/no outcomes, binomial regression will be used with a log link to give risk ratios. For other outcomes, where there is no baseline value, adjustment will be made for the primary outcome only. Analytes measured from a targeted NMR metabolome will be checked for normality; those with non-parametric distribution will be log-transformed. Analyte data at baseline and at the time of the diagnostic OGTT (24-28 weeks') will be compared between women who received the intervention and those who did not. To test for the effect of the intervention on dietary and physical activity outcomes, ANCOVA will be used adjusted for trial entry measurements. 


\section{Data monitoring}

A trial steering committee will be established to oversee the conduct and progress of the trial. The terms of reference of the Trial Steering Committee have been developed separately.

All adverse events (AEs) and serious adverse events (SAEs) will be recorded from the time a participant is randomised into the trial. All SAEs, serious adverse reactions (SARs) or unexpected serious adverse reactions (SUSARs) in the lifestyle and metformin arm will be reported immediately by the Chief Investigator to the King's Health Partners Clinical Trial Office (KHPs-CTO) in accordance with the current Pharmacovigilance Policy.

\section{Data management}

Types of data collected in this study will include participant information (anonymised) on demography, medical and family history and current pregnancy health which will be collected at study entry. Information on dietary intake, physical activity and glucose homeostasis will be collected pre- and postintervention. Following delivery, information will be collected from maternal and neonatal medical records regarding health in late pregnancy, labour onset, mode of delivery and inpatient nights, admission to special care baby unit. Neonatal outcome data will include admission to special care baby unit. A study specific web-based data platform (MedSciNet ${ }^{\mathrm{T}}$ ) will be used in this trial. All data entries will be logged, providing an effective audit trail including randomisation history. All records will be kept in line with applicable national laws and regulations.

\section{Confidentiality}

All data from maternity records, questionnaires and visit notes will be anonymised, kept confidential and stored in line with the Medicines for Human Use (Clinical Trials) Amended Regulations 2006 and the General Data Protection Regulation and archived in accordance with the Medicines for Human Use (Clinical Trials) Amended Regulations 2006. The trial database will be locked before the final analysis in line with the local guidelines. All anonymised data will be stored on a password protected computer with limited access. No personally identifiable information will be included in any publications or presentations relating to this study.

\section{Discussion}

Several RCTs including UPBEAT have attempted to improve pregnancy outcomes using lifestyle and/or pharmacological intervention in pregnant women with obesity. The lack of success of these RCTs in unselected women has shifted the focus to targeted interventions for those individuals identified at greatest risk of adverse pregnancy outcomes. Early identification and treatment of high-risk women may improve the health of the mother and her infant.

This study is designed to provide evidence that early risk stratification of pregnant women with obesity for later development of GDM and early targeted treatment using lifestyle advice with or without 
metformin improves glucose tolerance compared to standard antenatal care. If the study proves effective, the next stage will be the development of a protocol for an RCT adequately powered for examining a reduction in prevalence of GDM. The aim will be to determine, whether in a high-risk subgroup of pregnant women with obesity, targeted intervention with lifestyle advice +/- metformin is an effective strategy for prevention of GDM.

\section{Abbreviations}

\begin{tabular}{|ll|}
\hline AEs & Adverse events \\
\hline AUC & Area under the curve \\
\hline BMI & Body mass index \\
\hline CGM & Continuous glucose monitoring \\
\hline CV & Coefficient of variation \\
\hline FFQ & Food frequency questionnaire \\
\hline GDM & Gestational diabetes mellitus \\
\hline GI & Glycaemic index \\
\hline GL & Glycaemic load \\
\hline HBGI & High Blood glucose index \\
\hline IADPSG & The International Association of Diabetes and Pregnancy Study Groups \\
\hline KHPs-CTO & King's Health Partners Clinical Trial Office \\
\hline LBGI & Low Blood glucose index \\
\hline LGA & Large-for-gestational age \\
\hline NHS & The National Health Service \\
\hline NICE & The National Institute of Clinical Excellence \\
\hline NMR & Nuclear magnetic resonance \\
\hline OGTT & Oral glucose tolerance test \\
\hline RCT & Randomised controlled trials \\
\hline SAEs & Serious adverse events \\
\hline SARs & Serious adverse reactions \\
\hline SD & Standard deviation \\
\hline SUSARs & Unexpected serious adverse reactions \\
\hline
\end{tabular}




\section{Declarations}

\section{Funding}

This study is funded by the Wellcome Trust and Rosetrees Trust. SLW and LP are supported by Tommys charity. OFQ is supported by King Abdulaziz University, Jeddah, Saudi Arabia. PTS is partly funded by Tommy's and by ARC South London (NIHR).

\section{Authors' contributions}

ACF, SLW, LP were responsible for designing the study. OFQ prepared the first draft of the manuscript. Successive drafts were contributed by OFQ, ACF, SLW, LP, GN, JH, PTS, CG, AB, OF, IV, OO, EC, JP, KGN. The final draft of the manuscript was approved by all authors.

\section{Acknowledgements}

Not applicable.

\section{Availability of data and materials}

Not applicable.

\section{Ethics approval and consent to participate}

UK IRAS integrated research application system; reference 18/LO/1500.

\section{Consent for publication}

Not applicable.

\section{Competing interests}

Not applicable.

\section{References}

1. NCD Risk Factor Collaboration (NCD-RisC). Trends in adult body-mass index in 200 countries from 1975 to 2014: a pooled analysis of 1698 population-based measurement studies with $19 \cdot 2$ million participants NCD Risk Factor Collaboration (NCD-RisC)*. 2016.

2. National Maternity and Perinatal Audit. National Maternity and Perinatal Audit (NMPA) [Internet]. 2019. Available from: https://www.hqip.org.uk/resource/national-maternity-and-perinatal-auditnmpa-clinical-report-2019/\#.YRKR5NNKigQ

3. Poston L, Caleyachetty R, Cnattingius S, Corvalán C, Uauy R, Herring S, et al. Preconceptional and maternal obesity: epidemiology and health consequences. Lancet Diabetes Endocrinol. Lancet 
Publishing Group; 2016;4:1025-36.

4. Torloni MR, Betrán AP, Horta BL, Nakamura MU, Atallah AN, Moron AF, et al. Prepregnancy BMI and the risk of gestational diabetes: A systematic review of the literature with meta-analysis: Diagnostic in Obesity and Complications. Obes Rev. John Wiley \& Sons, Ltd; 2009;10:194-203.

5. Marchi J, Berg M, Dencker A, Olander EK, Begley C. Risks associated with obesity in pregnancy, for the mother and baby: A systematic review of reviews. Obes Rev. Blackwell Publishing Ltd; 2015;16:621-38.

6. Bryson CL, loannou GN, Rulyak SJ, Critchlow C. Association between Gestational Diabetes and Pregnancy-induced Hypertension. Am J Epidemiol. 2003;158:1148-53.

7. Billionnet C, Mitanchez D, Weill A, Nizard J, Alla F, Hartemann A, et al. Gestational diabetes and adverse perinatal outcomes from 716,152 births in France in 2012. Diabetologia. Springer Verlag; 2017;60:636-44.

8. Noctor E. Type 2 diabetes after gestational diabetes: The influence of changing diagnostic criteria. World J Diabetes. Baishideng Publishing Group Inc.; 2015;6:234.

9. Kramer CK, Campbell S, Retnakaran R. Gestational diabetes and the risk of cardiovascular disease in women: a systematic review and meta-analysis. Diabetol 2019626 [Internet]. Springer; 2019 [cited 2021 Jul 27];62:905:914. Available from: https://link.springer.com/article/10.1007/s00125-0194840-2

10. Hopmans TEP, van Houten C, Kasius A, Kouznetsova OI, Nguyen LA, Rooijmans S V., et al. Increased risk of type II diabetes mellitus and cardiovascular disease after gestational diabetes mellitus: a systematic review. Ned Tijdschr Geneeskd. 2015;159:A8043.

11. Young BC, Ecker JL. Fetal macrosomia and shoulder dystocia in women with gestational diabetes: Risks amenable to treatment? Curr Diab Rep. 2013;13:12-8.

12. Wang J, Wang L, Liu H, Zhang S, Leng J, Li W, et al. Maternal gestational diabetes and different indicators of childhood obesity: A large study. Endocr Connect. BioScientifica Ltd.; 2018;7:1464-71.

13. Blotsky AL, Rahme E, Dahhou M, Nakhla M, Dasgupta K. Gestational diabetes associated with incident diabetes in childhood and youth: A retrospective cohort study. CMAJ. Canadian Medical Association; 2019;191:E410-7.

14. Clausen TD, Mathiesen ER, Hansen T, Pedersen O, Jensen DM, Lauenborg J, et al. High prevalence of type 2 diabetes and pre-diabetes in adult offspring of women with gestational diabetes mellitus or type 1 diabetes: The role of intrauterine hyperglycemia. Diabetes Care. Diabetes Care; 2008;31:3406.

15. Ruchat SM, Houde AA, Voisin G, St-Pierre J, Perron P, Baillargeon JP, et al. Gestational diabetes mellitus epigenetically affects genes predominantly involved in metabolic diseases. Epigenetics. Taylor and Francis Inc.; 2013;8:935-43.

16. Pathirana MM, Lassi ZS, Ali A, Arstall MA, Roberts CT, Andraweera PH. Association between metabolic syndrome and gestational diabetes mellitus in women and their children: a systematic 
review and meta-analysis. Endocrine [Internet]. Springer; 2020 [cited 2021 Aug 10];71:310-20. Available from: https://doi.org/10.1007/s12020-020-02492-1

17. National Institute for Health and Care Excellence. Diabetes in pregnancy: management from preconception to the postnatal period. NICE. 2015.

18. Sovio U, Murphy HR, Smith GCS. Accelerated fetal growth prior to diagnosis of gestational diabetes mellitus: A prospective cohort study of nulliparous women. Diabetes Care. American Diabetes Association Inc.; 2016;39:982-7.

19. White SL, Pasupathy D, Sattar N, Nelson SM, Lawlor DA, Briley AL, et al. Metabolic profiling of gestational diabetes in obese women during pregnancy. Diabetologia. Springer Verlag; 2017;60:1903-12.

20. Mokkala K, Vahlberg T, Pellonperä O, Houttu N, Koivuniemi E, Laitinen K. Distinct Metabolic Profile in Early Pregnancy of Overweight and Obese Women Developing Gestational Diabetes. J Nutr. Oxford University Press; 2020;150:31-7.

21. The International Weight Management in Pregnancy (i-WIP) Collaborative Group. Effect of diet and physical activity based interventions in pregnancy on gestational weight gain and pregnancy outcomes: meta-analysis of individual participant data from randomised trials. BMJ. British Medical Journal Publishing Group; 2017;358:j3119.

22. Chiswick C, Reynolds RM, Denison F, Drake AJ, Forbes S, Newby DE, et al. Effect of metformin on maternal and fetal outcomes in obese pregnant women (EMPOWaR): A randomised, double-blind, placebo-controlled trial. Lancet Diabetes Endocrinol. Lancet Publishing Group; 2015;3:778-86.

23. Dodd JM, Louise J, Deussen AR, Grivell RM, Dekker G, McPhee AJ, et al. Effect of metformin in addition to dietary and lifestyle advice for pregnant women who are overweight or obese: the GRoW randomised, double-blind, placebo-controlled trial. Lancet Diabetes Endocrinol. Lancet Publishing Group; 2019;7:15-24.

24. Syngelaki A, Nicolaides KH, Balani J, Hyer S, Akolekar R, Kotecha R, et al. Metformin versus placebo in obese pregnant women without diabetes mellitus. N Engl J Med. Massachussetts Medical Society; 2016;374:434-43.

25. Koivusalo SB, Rönö K, Klemetti MM, Roine RP, Lindström J, Erkkola M, et al. Gestational Diabetes Mellitus Can Be Prevented by Lifestyle Intervention: The Finnish Gestational Diabetes Prevention Study (RADIEL): A Randomized Controlled Trial. Diabetes Care. American Diabetes Association Inc.; 2016;39:24-30.

26. H. Al Wattar B, Dodds J, Placzek A, Beresford L, Spyreli E, Moore A, et al. Mediterranean-style diet in pregnant women with metabolic risk factors (ESTEEM): A pragmatic multicentre randomised trial. Persson LÅ, editor. PLOS Med. Public Library of Science; 2019;16:e1002857.

27. Guo F, Yang S, Zhang Y, Yang X, Zhang C, Fan J. Nomogram for prediction of gestational diabetes mellitus in urban, Chinese, pregnant women. BMC Pregnancy Childbirth. BioMed Central Ltd.; 2020;20:43. 
28. Harrison CL, Lombard CB, East C, Boyle J, Teede HJ. Risk stratification in early pregnancy for women at increased risk of gestational diabetes. Diabetes Res Clin Pract. Elsevier Ireland Ltd; 2015;107:618.

29. Nanda S, Savvidou M, Syngelaki A, Akolekar R, Nicolaides KH. Prediction of gestational diabetes mellitus by maternal factors and biomarkers at 11 to 13 weeks. Prenat Diagn. 2011;31:135-41.

30. Abell SK, Shorakae S, Boyle JA, De Courten B, Stepto NK, Teede HJ, et al. Role of serum biomarkers to optimise a validated clinical risk prediction tool for gestational diabetes. Aust New Zeal J Obstet Gynaecol. Blackwell Publishing; 2019;59:251-7.

31. Donovan BM, Breheny PJ, Robinson JG, Baer RJ, Saftlas AF, Bao W, et al. Development and validation of a clinical model for preconception and early pregnancy risk prediction of gestational diabetes mellitus in nulliparous women. PLoS One. Public Library of Science; 2019;14:e0215173e0215173.

32. Sweeting AN, Appelblom H, Ross GP, Wong J, Kouru H, Williams PF, et al. First trimester prediction of gestational diabetes mellitus: A clinical model based on maternal demographic parameters. Diabetes Res Clin Pract. Elsevier Ireland Ltd; 2017;127:44-50.

33. Thériault S, Forest JC, Massé J, Giguère Y. Validation of early risk-prediction models for gestational diabetes based on clinical characteristics. Diabetes Res Clin Pract. Elsevier Ireland Ltd; 2014;103:419-25.

34. Poston L, Bell R, Croker H, Flynn AC, Godfrey KM, Goff L, et al. Effect of a behavioural intervention in obese pregnant women (the UPBEAT study): A multicentre, randomised controlled trial. Lancet Diabetes Endocrinol. Lancet Publishing Group; 2015;3:767-77.

35. White SL, Lawlor DA, Briley AL, Godfrey KM, Nelson SM, Oteng-Ntim E, et al. Early Antenatal Prediction of Gestational Diabetes in Obese Women: Development of Prediction Tools for Targeted Intervention. Samocha-Bonet D, editor. PLoS One. Public Library of Science; 2016;11:e0167846.

36. Chan AW, Tetzlaff JM, Altman DG, Laupacis A, Gøtzsche PC, Krleža-Jerić K, et al. SPIRIT 2013 statement: Defining standard protocol items for clinical trials. Ann Intern Med. American College of Physicians; 2013;158:200-7.

37. International Association of Diabetes and Pregnancy Study Groups Consensus Panel. International Association of Diabetes and Pregnancy Study Groups recommendations on the diagnosis and classification of hyperglycemia in pregnancy. Diabetes Care. 2010;33:676-82.

38. Yogev Y, Ben-Haroush A, Chen R, Rosenn B, Hod M, Langer O. Diurnal glycemic profile in obese and normal weight nondiabetic pregnant women. Am J Obstet Gynecol. 2004;191:949-53.

39. Maitland R, Patel N, Barr S, Sherry C, Marriage B, Seed P, et al. A Slow-Digesting, Low-Glycemic Load Nutritional Beverage Improves Glucose Tolerance in Obese Pregnant Women Without Gestational Diabetes. Diabetes Technol Ther. Mary Ann Liebert Inc.; 2018;20:672-80.

40. Harmon KA, Gerard L, Jensen DR, Kealey EH, Hernandez TL, Reece MS, et al. Continuous glucose profiles in obese and normal-weight pregnant women on a controlled diet: Metabolic determinants of fetal growth. Diabetes Care. 2011;34:2198-204. 
41. Vickers AJ, Altman DG. Analysing controlled trials with baseline and follow up measurements. BMJ [Internet]. British Medical Journal Publishing Group; 2001 [cited 2021 Aug 3];323:1123-4. Available from: https://www.bmj.com/content/323/7321/1123

\section{Figures}

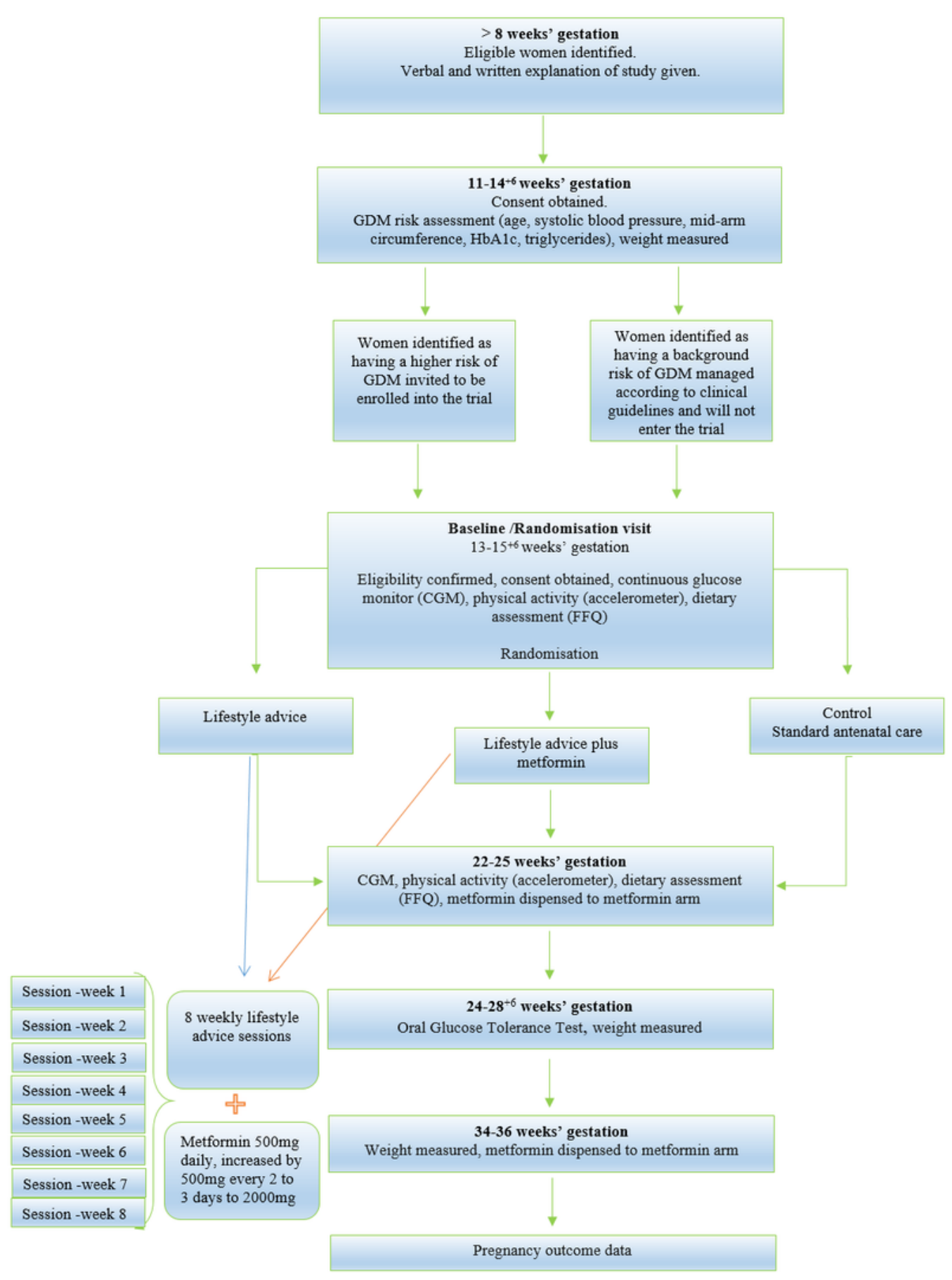


CONSORT study flow diagram for UPBEAT-TIF pilot study 